\title{
Coulisses
}

Revue de théâtre

42 | Printemps 2011

Racine : Théâtre et émotion

\section{Athalie ou le dénouement de la sorcellerie}

\section{Normand Doiron}

\section{OpenEdition}

Journals

Édition électronique

URL : https://journals.openedition.org/coulisses/578

DOI : 10.4000/coulisses.578

ISSN : 2546-9460

\section{Éditeur}

Presses universitaires de Franche-Comté

\section{Édition imprimée}

Date de publication : 15 février 2011

Pagination : $31-41$

ISBN : 978-2-84867-316-5

ISSN : 1150-594X

\section{Référence électronique}

Normand Doiron, «Athalie ou le dénouement de la sorcellerie », Coulisses [En ligne], 42 | Printemps

2011, mis en ligne le 30 novembre 2016, consulté le 29 décembre 2022. URL : http://

journals.openedition.org/coulisses/578; DOI : https://doi.org/10.4000/coulisses.578 


\title{
Athalie ou le dénouement de la sorcellerie
}

\author{
NORMAND DOIRON \\ Université McGill, \\ Montréal
}

\begin{abstract}
3 Witch - All hail, Macbeth, that shalt be king hereafter ${ }^{1}$ !

Shakespeare, Macbeth, I, 3.
\end{abstract}

\section{La première tragédie}

La tragédie, en France, naît, s'épanouit et, peut-être avec Athalie, atteint son plus haut degré sous le signe de la magie. Par ses péripéties funestes et ses piteuses catastrophes, par les passions qu'elle allume et qu'elle attise sous le prétexte de les purger, par les critiques virulentes qu'en fait l'Église sans discontinuer, la tragédie est liée, on le sait, au mal, au malheur, à la malédiction, comme celle qui pèse sur les Atrides, mais encore aux maléfices, au malin. La tragédie, telle qu'elle apparaît au milieu du XVI ${ }^{e}$ siècle, est diabolique par nature, parce que le diable est le maitre de tous les masques, de tous les déguisements et de tous les mensonges. Il joue chaque fois un nouveau rôle mais, Chrysostome ou Augustin le clamaient en condamnant les spectacles, il suscite toujours la même concupiscence. C'est l'origine morale du théâtre, considéré d'un point de vue diabolique.

Mais les racines du mal sont politiques. L'État monarchique ne prend «sur les consciences" un tel pouvoir "qu'en dramatisant à l'excès la figure du diable $»^{2}$. Couronné, Lucifer domine de toute sa puissance la horde des démons qu'on voyait indisciplinés et burlesques dans les diableries médiévales. Ainsi

1. La troisième sorcière - «Salut, Macbeth, salut à toi, qui un jour seras roi!» (trad. JeanClaude Sallé).

2. Robert Muchembled, Le roi et la sorcière. L'Europe des bûchers (XVe-XVIII siècle), Paris, Desclée, 1993, p. 44. 
que le roi dans son royaume, il détient dans l'empire du péché une autorité souveraine. Ce prince des ténèbres, ou bien ceux qui le représentent sur terre, les sorciers, mieux encore les sorcières, les reines dans le mal comme Athalie, figurent exactement l'envers du monarque armé de lumière.

Or, devenant administrateur, perdant avec la centralisation de l'État une grande part de ses attributs religieux, «le roi devient prêtre en représentation ». Dans l'État moderne, seule la représentation permet d'assurer «la réconciliation du rex et du sacerdos $»^{3}$. Par le spectacle, le roi retrouve les forces sacrées qui fondaient son pouvoir, alors que dans la bataille il exposait sa vie à la justice de Dieu. Aussi, avant même d'être le lieu de sa conquête de l'espace politique, la tragédie est-elle celui de sa consécration par sa lutte contre les anges du mal. Il n'est pas question de savoir si un roi de théâtre l'emporte ou non sur les puissances de l'illusion, mais de constater que le monarque réel, en raison des pouvoirs magiques que lui confère la scène, apparaît comme seul capable de défendre son royaume contre les forces maléfiques. La relation du politique et du religieux est précisément le sujet d'Athalie. Comme si Racine, loin de rompre avec la tragédie, renouait avec ses origines.

La Cléopatre captive de Jodelle, jouée devant le roi et sa cour (1553), est en général considérée comme la première tragédie française. Mais La Médée de La Péruse, quand elle parait en 1556 à Poitiers, est la première tragédie française qui ait jamais été imprimée 4 . Ce n'est pas un mince titre de gloire pour le jeune La Péruse. Qu'il imite Euripide et Sénèque ne dégarnit pas son front, que tous les poètes du $\mathrm{XVI}^{\mathrm{e}}$ siècle ont orné de lauriers. Car du premier coup il met en scène les deux personnages dont l'affrontement allait constituer le nœud de la tragédie moderne : la magicienne et le roi, Médée bravant Créon. Or, dans Médée, le roi subit le supplice que, dans les faits, subissent les sorcières au même moment, partout en Europe. Comme si La Péruse, non sans témérité, présentait au prince une espèce de miroir où les rôles sont inversés.

À Médée dans l'Antiquité classique, correspond Jézabel, la mère d'Athalie dans l'Ancien Testament. La reine idolâtre du Second Livre des Rois rivalise de cruauté avec la sorcière lascive de la mythologie. Elles arrivent en tête du répertoire des femmes féroces et assassines, ainsi que le dressent les recueils d'estampes, les livres d'emblèmes et les Figures de la Bible 5 .

3. Jean-Marie Apostolidès, Le prince-sacrifié. Théâtre et politique au temps de Louis XIV, Paris, Minuit, 1985, p. 25.

4. Jean de La Péruse, La Médée [1556], éd. par James A. Coleman, University of Exeter, 1985; La Médée [s.d.], éd. par Michel Dassonville, dans La tragédie à l'époque d'Henri II et de Charles IX, Florence, Leo S. Olschki, Paris, Presses Universitaires de France, 1989.

5. Sara F. Matthews Grieco, Ange ou diablesse. La représentation de la femme au XVTe siècle, Paris, Flammarion, 1991, p. 346-356. Georges Couton, «Athalie ou la leçon d'Histoire Sainte », dans Prémices et floraison de l'Âge classique. Mélanges en l'bonneur de Jean Jehasse, Publications de l’Université de Saint-Étienne, 1995, p. 425-440. 
Du reste, même la Cléopâtre captive n'est pas sans lien avec la magie. L'ombre d'Antoine, qui occupe le prologue, hante toute cette tragédie, attire son amante vers l'abîme, exerce sur elle un charme morbide. Il est faux que les ombres, dans les tragédies humanistes, ne jouent qu'un rôle de convention, hérité du théâtre sénéquien. Elles prennent dans les tragédies des $\mathrm{XVI}^{\mathrm{e}}$ et $\mathrm{XVII}{ }^{\mathrm{e}}$ siècles, une fonction dramaturgique originale. D'emblée elles installent la scène dans un au-delà qui donne à la représentation une étrange profondeur, qui confère aux personnages une existence fantomatique redoublant l'illusion théâtrale, et qui investit l'action d'une signification occulte, proche de l'allégorie.

La pièce de La Péruse n'est pas tant «première » dans l'ordre chronologique, que principe dans l'ordre esthétique. Elle donne le modèle d'un face-àface du pouvoir magique et du pouvoir politique. Médée représente l'envers terrifiant, l'ombre du prince qui par sa victoire sur les puissances infernales légitimera son droit divin. La sorcière exhibe la figure grimaçante de la méchanceté à laquelle se heurte celui qui règne.

Bien qu'il ignore complètement La Péruse, Pierre Corneille écrit non seulement, en début de carrière, une Médée (1635), mais encore, au sommet de sa gloire, une "pièce à machines », La Conquête de la Toison d'or (1660), dans laquelle se retrouve « la fille d'Aète, amante de Jason ». Dès la version d'Euripide, la tragédie de Médée supposait l'intervention d'un deus ex machina, puisqu'elle se terminait par la fuite scandaleuse de la régicide et de l'infanticide sur un char traîné par des dragons ailés. La magicienne appelle les machines dont les effets spectaculaires, au XVII ${ }^{\mathrm{e}}$ siècle, tiennent eux-mêmes de la magie. Sur la musique de Marc-Antoine Charpentier, Thomas Corneille écrit une Médée qui paraît en 1693. L’année suivante, paraît la Médée de Longepierre. Athalie est publiée en 1691.

Une autre tragédie humaniste, cette fois d'inspiration biblique, sert de modèle pour la confrontation de la sorcière et du roi : c'est Saül. Premier roi d'Israël, monarque à peine indépendant de la caste religieuse que représente le prophète Samuel (mort avant que ne commence l'action), Saül n'assume pas son pouvoir. Désespéré, il consulte une nécromancienne. Elle doit tirer de l'audelà Samuel dont il attend de l'aide.

Le sujet de cette tragédie, directement lié à la fameuse scène du songe d'Athalie, fut repris en Europe par de très nombreux imitateurs ${ }^{6}$. En France, Jean de la Taille, le premier, publie un Saül (1572), précédé d'un Art de la tragédie qui peut se lire, pour une part, comme une poétique de la lutte entre les rois et les mauvais sorts. Pierre Du Ryer publie en 1647 un Saül très ambigu. La scène de nécromancie soulevait une importante question : est-ce vraiment l'âme de

6. Pour la période allant du XVe au milieu du XIX ${ }^{\mathrm{e}}$ siècle, François Lecercle a identifié 75 adaptations pour la scène, opéras compris. Il en donne la liste dans «Saül et les effets de spectacle ", Les tragédies de Jean de La Taille, Cabiers Textuel, n¹8, 1998, p. 35-42. 
Samuel qui répond à l'appel, ou l'apparition, sous les dehors du prophète, n'estelle qu'une illusion diabolique? Chaque version de Saül propose une réponse originale, qui démontre que les auteurs connaissaient les enjeux théologiques du sujet. La tragédie devient une mise en scène de la démonologie ${ }^{7}$.

Saül et Médée suffisent à montrer que le roi et la sorcière constituent, dans leur antagonisme, l'un des puissants ressorts de la tragédie ${ }^{8}$.

\section{La dernière sorcière}

Racine, il n'y a pas de doute, trace en décrivant Athalie le portrait d'une sorcière. Il la saisit dans cette intenable situation où se trouvait Médée. De même que la magicienne de Colchide affrontait Créon, Athalie affronte Joas.

Plusieurs critiques et Racine lui-même ont utilisé un vocabulaire très suggestif, sans affirmer pourtant qu'Athalie était une sorcière. Le poète la dit blasphématrice, sacrilège et impie. "Cette femme $[\ldots]$ a souillé les regards ${ }^{9}$ : expression qui joint au mauvais œil l'idée religieuse du miasme ou de la tache. La thématique d'une obscurité sacrée traverse toute la tragédie. Dès les premiers vers, Athalie transforme les beaux jours d'autrefois «en des jours ténébreux $»^{10}$. Abner inquiet est agité d'un «noir pressentiment». Il redoute le «sombre chagrin» qui accable Athalie et la «noirceur» du fiel que distille Mathan. "Une profonde nuit $»^{11}$ ouvre le songe horrible, que la reine voudrait réduire à l'effet de quelque «sombre vapeur». Le monde occulte se manifeste encore, de manière plus menaçante, dans le schéol rempli de flammes qui s'ouvre sous les pieds du diabolique Mathan. Joad s'inquiète pour son épouse :

Vous souffrez qu'il vous parle ! et vous ne craignez pas

Que du fond de l'abîme entr'ouvert sous ses pas

Il ne sorte à l'instant des feux qui vous embrasent ${ }^{12}$ ?

Mathan est ministre du culte de Baal. En tant qu'idolâtre, il n'en faut pas plus, Athalie s'est donnée au diable. Le «pacte avec l'impiété »"13, que dénonce Joad,

7. François Lecercle, «La démonologie en scène. À propos des versions théâtrales de l'Épisode d'Endor (I Rois / Samuel, 28) ", dans Françoise Lavocat, Pierre Kapitaniak et Marianne Closson, dir., Fictions du diable. Démonologie et littérature de saint Augustin à Léo Taxil, Genève, Droz, 2007, p. 169-199. Voir aussi N. Doiron, "Saül sur la scène », dans Études françaises, 2008, vol. 44, n², p. 33-50.

8. On trouvera d'autres exemples dans l'excellente thèse d'Aurore Gutierrez Laffond, Théatre et magie dans la littérature dramatique du XVII siècle en France, Lille, Atelier National de Reproduction des Thèses, 1998.

9. Racine, Athalie, v. 747-748.

10. Athalie, v. 14.

11. Athalie, v. 490.

12. Athalie, v. 1021-1023.

13. Athalie, v. 90. 
rappelle, en bonne démonologie, le contrat que les sorciers passent avec le diable et qui peut d'ailleurs servir contre eux dans les procès ${ }^{14}$.

Les critiques, en général, ne dépassent pas l'allusion. Pour Roland Barthes, Athalie est une «exploratrice de secrets interdits » ${ }^{15}$. Pour Constant Veneosen, Athalie est un "personnage maudit». Par sa « révolte contre le vrai Dieu », elle annonce un «Mal satanique». Mathan possède de même "une stature démoniaque étonnante ${ }^{16}$. Paul Pelckmans parle de Jezabel comme d'une « reine damnée ${ }^{17}$. Seul Charles Mauron affirme sans détour qu'Athalie est une sorcière : «À l'époque de Bajazet, l'idée ne semble pas être née que le fils puisse, avec l'aide du père, retourner l'agressivité contre la sorcière - ce qu'Athalie marquera si clairement $»^{18}$. La sorcière apparaît sous la femme, lorsqu'une figure maternelle appelle «amour ce qui est possession». Agrippine, Clytemnestre, Phèdre comptent parmi les ancêtres d'Athalie. De sorte que la figure de la sorcière traverse l'œuvre de Racine. Dans ce terme de possession, la pulsion psychologique croise le pouvoir diabolique. C'est là, en ce point où l'amour sans frein rencontre la sorcellerie, que nous trouvons Médée.

Ce qui fait d'Athalie une sorcière, une parente de Médée dans le crime, c'est d'abord l'infanticide. Elle est « sanguinaire ${ }^{19}$. Elle massacre ses petits-enfants et c'est par miracle que Joas échappe à la mort. Il grandit caché dans le temple. Bien que l'usurpatrice ignore qu'un héritier légitime du trône a survécu, toute la tragédie tourne autour du danger, achevant dans le sang de son dernier fils la tuerie qu'elle avait si bien commencée. Quand, charmée par sa douceur et sa grâce, Athalie veut adopter Joas - qu'elle n'a pas reconnu comme son propre fils - elle ne fait que changer de méthode : elle veut avaler celui qu'elle n'a pu égorger. Elle veut enfoncer dans son sein celui qui la poignarde en rêve. La sorcière est une ogresse. Le cannibalisme est l'une des voies diaboliques de l'infanticide.

Comme Médée était barbare, Athalie est " étrangère $»^{20}$, le chœur le chante en refrain. Il ne s'agit pas d'un vague rapprochement. Dans son commentaire à l'édition française des Livres des Rois (1674), Le Maître de Sacy avait explicite-

14. On sait que Sigmund Freud accorde beaucoup d'importance à ce motif du pacte: «Une névrose diabolique au XVII siècle» [1923], dans L'inquiétante étrangeté et autres essais, Paris, Gallimard, 1985, p. 269-315. Michel de Certeau décrit les pactes comme des preuves objectives ayant joué un rôle crucial dans le procès d'Urbain Grandier, dans La possession de Loudun, Paris, Gallimard, 2005, p. 183-193.

15. Roland Barthes, Sur Racine, Paris, Éditions du Seuil, 1963, p. 125.

16. Constant Venesoen, Jean Racine et le procès de la culpabilité, Paris, La Pensée Universelle, 1981, p. 211, p. 215, p. 224, p. 227.

17. Paul Pelkmans, Le rêve apprivoisé. Pour une psychologie historique du topos prémonitoire, Amsterdam, Rodopi, 1986, p. 118.

18. Charles Mauron, L'inconscient dans l'auvre et la vie de Racine, Aix-en-Provence, Ophrys, 1957, p. 106.

19. Athalie, v. 59.

20. Athalie, v. 799. 
ment comparé les deux sorcières, insistant sur la possession : «D’autres auteurs disent qu'Athalie étant d'un naturel très violent, et possédée du désir de régner seule dans Juda, se porta à cet excès de fureur, ainsi qu'une autre Médée, que de tuer ses enfants, c'est-à-dire ses petits-enfants, pour s'assurer la couronne par le meurtre $»^{21}$.

Plusieurs éléments permettent de regarder le «songe» d'Athalie comme une vision diabolique. Mais le plus important d'entre eux reste que son «âme » en est "possédée $»^{22}$. Le fait que la sorcière elle-même parle d'un "songe» n'enlève rien au diable. On connait les débats sur la question de savoir si la sorcière se rendait physiquement sur les lieux du sabbat, ou bien si, croyant voler sur son balai, elle restait en réalité dans son lit, ne baisant qu'en rêve le cul du bouc. Dans ce dernier cas, celui de l'hallucination, le songe lui-même devenait sorcellerie, «comme expérience onirique induite par le diable $»^{23}$.

Le songe commence soudainement, par une rupture narrative marquée même par le temps des verbes. Athalie n'aperçoit pas sa mère, Jézabel se jette devant sa fille : elle "se montre", surgissant de la nuit profonde. Ce fantôme "pompeusement paré $»^{24}$ possède tous les caractères d'une apparition diabolique. C'est l'un des tours du diable que de parader sous les traits d'une femme, qui n'est plus l'instant d'après qu'une immonde charogne ${ }^{25}$.

Athalie parle de l'« ombre » de sa mère se baissant vers son lit. Est-il possible qu'une âme revienne sur terre, qu'elle se montre et qu'elle parle ? La réponse est claire, dans le cas d'une âme damnée: non, elle ne peut d'aucune manière sortir de la prison éternelle où elle brûle. Le spectre ne serait donc qu'une ruse diabolique, une illusion qui effraie et qui trouble l'esprit. Et c'est bien ce que dit Abner, sans mesurer la menace que contiennent ses paroles : «De vos songes menteurs l'imposture est visible $»^{26}$. Joad demande à Dieu la chute d'Athalie, c'est ce que Dieu lui donnera par l'intermédiaire du diable.

Le mot «trouble» décrit mieux que tout autre le traumatisme du songe. C'est le premier dont use Athalie pour en parler : « un trouble importun ». C'est lui aussi qu'elle utilise pour clore sa tirade : «Voilà quel trouble ici m’oblige à m'arrêter $»^{27}$. C'est celui dont s'était servi Mathan pour l'inciter aux confi-

21. Cité par Georges Forestier, Jean Racine, Gallimard. 2006, p. 714.

22. Athalie, v. 519.

23. Stuart Clark, «Songes diaboliques et paradoxes visuels », dans Fr. Lavocat et al., dir., op. cit., p. 267.

24. Bossuet, Maximes et réflexions sur la comédie [1694], Paris, Aux Éditions du Raisin, 1929, p. 36 : « [Les Pères] blâment, dans les jeux et dans les théâtres, l'inutilité, la prodigieuse dissipation, le trouble, la commotion de l'esprit [...], les grands ornements, qu'ils mettent au rang des pompes que nous avons abjurées par le baptême».

25. Un exemple dans François de Rosset, Les histoires mémorables et tragiques de ce temps [1619], X, éd. par Anne de Vaucher Gravili, Paris, Librairie Générale Française, 1994, p. 259.

26. Athalie, v. 656.

27. Athalie, v. 541. 
dences : «Quel trouble vous agite, et quel effroi vous glace ? " ${ }^{28}$. C'est encore lui que choisira la reine pour décrire l'émotion qu'elle éprouve en présence de Joas : «Quel prodige nouveau me trouble et m'embarrasse ! ${ }^{29}$. Enfin, Joad est confiant en la force de son coup : «Déjà ce Dieu vengeur commence à la troubler $»^{30}$. Et c'est justement le mot qu'on trouve chez Pierre de Lancre, le tristement célèbre démonologue, quand il décrit les funestes effets d'un « Demon ou Spectre » : « cette vision apportoit du trouble $»^{31}$.

Mais ce trouble n'est que l'aspect psychologique du bouleversement général qui témoigne de l'action du diable. Les sorcières renversent l'ordre naturel des choses, elles brisent l'harmonie du monde. Robert Muchembled regarde la sorcellerie comme une «immense métaphore du désordre régnant alors sur l'ensemble d'une Europe fracturée et inquiète $»^{32}$. Là où se trouve Athalie, là survient le chaos. Dans le songe, c'est l'image de l'« horrible mélange / d'os et de chair ${ }^{33}$ en quoi se transforme subitement Jézabel ; c'est le « bizarre assemblage » des objets qu'elle vient de décrire. Pénétrant dans le temple, Athalie sème le «tumulte ${ }^{34}$, y jette un "désordre funeste ${ }^{35}$ dont Zacharie s'émeut. Nabal s'étonne de « cette confusion $»^{36}$ qui règne dans l'esprit de la reine. Bien plus encore il s'étonne de voir Mathan qui «se trouble » au point de ne plus trouver la sortie du temple : «De vos sens étonnés quel désordre s'empare ${ }^{37}$. Cette panique du prêtre cherchant une issue annonce déjà le piège qui se refermera sur Athalie. Le chœur déplore, à la fin du Troisième Acte, « le désordre extrême » dans lequel se prépare le couronnement de Joas. La sorcière est l'envers du roi. Plus profond était le gouffre où vivait l'idolâtre, plus grande sera l'élévation du monarque. Mais d'abord le désordre se propage partout, à mesure que le désarroi s'empare d'Athalie.

Ce qui fait d'elle une sorcière, c'est aussi la terrible malédiction, un moment avant sa mort, qu'elle prononce contre Joas :

On verra de David l'héritier détestable

Abolir tes honneurs, profaner ton autel,

Et venger Athalie, Achab et Jézabel ${ }^{38}$.

28. Athalie, v. 460.

29. Athalie, v. 651.

30. Athalie, v. 1343.

31. L'incrédulité et mescréance du sortilège, 1622, chap. iv, p. 369, cité par Pierre Kapitaniak, «Entre ruse diabolique et illusion dramatique. Cheminement du discours démonologique dans l'Angleterre jacobéenne », dans Fr. Lavocat et al., dir., op. cit., p. 210.

32. R. Muchembled, op. cit., p. 58.

33. Athalie, v. 503-504.

34. Athalie, v. 433.

35. Athalie, v. 420.

36. Athalie, v. 862.

37. Athalie, v. 1043.

38. Athalie, v. 1788-1790. 
Ces mauvais mots ont la force d'un envoûtement. Dans le monde de l'Ancien Testament "mal et malheur procèdent de la bouche des méchants", dont "les paroles étaient empoisonnées $»^{39}$. En principe, les élus de Yahvé ne pratiquaient pas la magie. Dans les faits, le Dieu Unique s'était assez mal dégagé de la foule des esprits et des démons qu'avait toujours fréquentés le peuple juif. Ainsi, on a pu voir « dans beaucoup de psaumes des prières demandant à Dieu de libérer ses fidèles des charmes des méchants $»^{40}$. La prière que prononce Joad est de ce type, c'est une imprécation faite au nom du Très-Haut. Invoquant le Bien tout-puissant, il veut le mal :

Daigne, daigne, mon Dieu, sur Mathan et sur elle

Répandre cet esprit d'imprudence et d'erreur,

De la chute des rois funestes avant-coureur ${ }^{41}$ !

Et ce vœu sera exaucé. Athalie tombera, parce que Joad a supplié Yahvé. La première conséquence de cet anathème jeté sur la reine, c'est précisément le songe dans lequel, de manière frappante, Jézabel répète la malédiction que vient de proférer Joad. Elle annonce la prochaine déchéance d'Athalie :

Tremble, m'a-t-elle dit, fille digne de moi ;

Le cruel Dieu des Juifs l'emporte aussi sur toi ${ }^{42}$.

Dans cette tragédie, résonnent des «mots épouvantables »" , qui agissent sur la matière et les esprits, des mots magiques qui nouent et qui dénouent.

La deuxième conséquence de l'anathème, c'est qu'Athalie, quand elle s'introduit dans le temple et qu'elle s'avance, prête à violer l'enceinte réservée aux lévites, tout à coup s'arrête, hypnotisée par le jeune Joas. Elle veut parler, ou du moins crier de surprise ou de peur, mais sa gorge s'est nouée : «Mais sa langue en sa bouche à l'instant s'est glacée $»^{44}$. Le mauvais sort jeté par le prêtre n'est pas sans effet: la reine est d'abord troublée, puis paralysée, enfin, c'est l'ultime conséquence, la reine est prise au piège et achevée : "Grand Dieu ! voici ton heure, on t’amène ta proie $! »^{45}$. Cette tragédie représente une véritable chasse. À la fois bouche pleine d'incantations et gueule carnassière, le temple se referme sur la reine. La meute de chiens ou de démons qui dévoraient sa mère est devenue pour Athalie une troupe de prêtres armés jusqu'aux dents. En pénétrant sans méfiance dans le sanctuaire, elle est attrapée, le songe lui-même n'était qu'un leurre, et Joas un appât.

39. Alfred Guillaume, Prophétie et divination chę les Sémites, Paris, Payot, 1950, p. 335-336.

40. Ibid., p. 325 .

41. Athalie, v. 292-294.

42. Athalie, v. 497-498.

43. Athalie, v. 500.

44. Athalie, v. 411.

45. Athalie, v. 1668. 
Mais le théâtre, en tant qu'illusion, n'est-il pas lui-même le plus dangereux des pièges? Certes Athalie n'est pas du théâtre, auquel Racine a renoncé avec Phèdre. Mais c'est tout de même Racine qui a provoqué l'un des plus âpres épisodes de la Querelle du théâtre, en répliquant à Nicole qui pourtant ne le visait pas. Dans sa Première Visionnaire, Nicole s'attaquait à Desmarets : "un poète de théâtre est un empoisonneur public, non des corps mais des âmes $»^{46}$. Ce n'est pas la gravité, mais les termes de l'accusation qui nous intéressent. Nicole aurait pu tuer autrement les victimes du théâtre, les pourfendre ou les brûler. Non, il les empoisonne, c'est-à-dire qu'il choisit de tuer ainsi que les sorcières le font de préférence. De Médée à la Jobin, le poison est l'arme de celles qui connaissent les forces occultes de la nature. Le plus souvent, le sang des jeunes enfants entre dans la fabrication de ces potions universellement meurtrières, qui enlèvent la vie aux hommes, aux bêtes et qui détruisent les moissons.

Nicole donc ne dénonce pas seulement la corruption morale qu'entraîne le théâtre, il va plus loin et surtout plus précisément. Il associe la scène à la sorcellerie et la fameuse purgation à l'empoisonnement. Bossuet, dans ses Maximes et réflexions sur la comédie, ne raisonne pas autrement, quand il examine comment un acteur s'y prend pour jouer avec naturel une passion : il doit « rappeler autant qu'il peut [les passions] qu'il a ressenties [...], il faut qu'elles lui reviennent avec tous leurs agréments empoisonnés et toutes leurs grâces trompeuses $»^{47}$. Quand son âme est ensorcelée par ses propres mouvements désordonnés, le comédien peut donner au public la drogue qu'il réclame. On voit que Bossuet est à l'opposé de l'esthétique que développera Diderot dans son Paradoxe sur le comédien. Pour ses détracteurs au XVII ${ }^{\mathrm{e}}$ siècle, le jeu ne comporte aucune distance critique. L'acteur est entièrement possédé par ses émotions ${ }^{48}$. Un peu plus loin dans son opuscule, Bossuet fait des comédiennes non plus des sorcières, mais des «Sirènes »; «leur venin » cependant reste le même : "elles reçoivent de tous côtés, par les applaudissements qu'on leur renvoie, le poison qu'elles répandent par leur chant $»^{49}$.

Aussi plusieurs condamnèrent Athalie, comme ils condamnaient en général la comédie. Louis XIV, et le roi et la reine d'Angleterre assistèrent à la troisième représentation. «Mais l'évêque de Chartres, dont dépendait Saint-Cyr, avait refusé l'invitation ». Il avait préféré dénoncer «ces mascarades scandaleuses et ces débauches effroyables aux yeux de Dieu $»^{50}$. Même avec ses chœurs, son temple, ses prêtres et ses lévites, Athalie restait une œuvre diabo-

46. [Pierre Nicole], «Lettre XI, ou Première Visionnaire », L'Hérésie imaginaire, Liège, 1667, p. 8.

47. Bossuet, op. cit., p. 17.

48. Jean Duvignaud, «Existence et possession », dans L'Acteur. Esquisse d'une sociologie du comédien, Paris, Gallimard, 1965, p. 244-269.

49. Ibid., p. 26.

50. Jean Rohou, Jean Racine, Athalie, Presses Universitaires de France, 2003, p. 101. 
lique, mais écrite, semblait-il, pour dénoncer l'illusion même qui la fonde et vaincre le malin d'où elle tire son pouvoir.

\section{Le roi et la sorcière}

Mathan est un rôle inventé par Racine. Il incarne le courtisan vil et sans scrupule. Devant Nabal, son confident, il montre son véritable et méprisable visage. Il retrace avec fierté sa honteuse carrière : la querelle qu'il eut avec Joad, ses brigues, sa dissimulation. De sorte que son «âme à la cour s'attacha toute entière $\aleph^{51}$. Pour un peu de pouvoir, l'apostat vendit son âme. Salomith et le chœur des jeunes filles reprendront, mais sur un autre ton, le portrait du courtisan. Elles justifient l'inaction d'Abner. Quand il le voudrait, que pourrait-il faire?

Hélas ! dans une cour où l'on n'a d'autres lois

Que la force et la violence,

Où les honneurs et les emplois

Sont le prix d'une aveugle et basse obéissance ${ }^{52}$.

Aussi bien dire qu'il n'y a pas de loi, sinon celle du plus fort. Joad n'aura de cesse d'opposer à cette forme d'anarchie, la sainte loi que représente et que défend le monarque. Il va jusqu'au bout de son mépris pour la cour, et c'est directement au roi qu'il adresse une solennelle mise en garde contre les « lâches flatteurs ». Le roi doit se défier de ceux qui lui parlent d'une "voix enchanteresse ", il doit se défier du trône lui-même et de son "charme empoisonneur $\aleph^{53}$. Le vocabulaire de la sorcellerie sert à noircir les courtisans qui n'hésitent pas, pour satisfaire leurs vices, à entraîner le monarque avec eux dans l'abîme. Ils voudront le convaincre, renversement typique de la sorcellerie, que

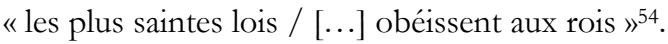

Si le poison est le symbole de la sorcière, le trésor est celui du courtisan. La reine, aveuglée par la convoitise, fait irruption avec des soldats dans le temple. Mais c'est Mathan qui avait fait miroiter devant ses yeux des richesses imaginaires, prétexte à saccager le temple. Dans les conseils qu'il donne à Joas, au moment de le couronner, Joad prend bien soin de souligner qu'un roi : «Sur la richesse et l'or ne met point son appui $»^{55}$. La fameuse toison, pour laquelle en Colchide Jason abattit le dragon, est le symbole de la royauté, et non pas du luxe et de l'opulence.

51. Athalie, v. 932.

52. Athalie, v. 1199-1202.

53. Athalie, v. 1390 , v. 1388.

54. Athalie, v. 1391-1392.

55. Athalie, v. 1279. 
La sorcellerie, la cour et le théâtre se fondent en une seule et puissante menace. Le roi, pour la contrer, possède la loi. Elle est omniprésente, dans cette tragédie qui se déroule au cours de : «La fameuse journée / Où sur le mont Sina la loi nous fut donnée $»^{56}$. Le chœur chante : "Ô divine, ô charmante loi $»^{57}$. Que répond Joas, lorsque Athalie lui demande son emploi ? "J'adore le seigneur; on m'explique sa loi $\aleph^{58}$. Et les derniers mots, prononcés par Joad, sont pour dire : «Que les rois dans le ciel ont un juge sévère " $^{9}$.

Cette loi, pour le peuple juif, est entièrement contenue dans «le livre redoutable $»^{60}$. Droit civil et droit religieux se confondent pour nous ramener à l'origine mythique du droit divin. Mais la victoire sur le mal est provisoire. Sous savons déjà, par la malédiction proférée par Athalie, que Joas trahira le Dieu qui l'a sacré. De sorte que nous assistons, et sans doute est-ce la véritable tragédie, à la division interne de la loi, à la naissance de l'histoire se séparant de l'Éternité maternelle toujours tentée de la tuer. D'un côté, les cupides révèlent au roi la nature profane des lois qu'il promulgue et qu'il garde. De l'autre, la magicienne lui rappelle la nature sacrée de la loi primitive.

En face de la loi sainte, il y a tous les hommes de loi qui constituèrent le plus ferme appui de l'État moderne centralisateur. Il y a toute la culture judiciaire, Bodin en tête, qui formula l'idée que la sorcellerie était un crime de lèsemajesté divine. C'est la justice laïque qui poursuit les sorcières. "L'histoire des bûchers est d'abord celle de la loi » ${ }^{61}$.

D’une part, comme Jason à travers Médée, Joas conquiert à travers Athalie le pouvoir magique dont il avait besoin pour achever sa quête. D'autre part, l'horreur de la sorcellerie dénonce les limites du pouvoir royal et les soubassements ignobles de la royauté. Au dénouement, la sorcière condamnée crie qu'elle revivra à travers celui qui l'exécute. Aussi longtemps qu'il y aura des rois, il y aura des sorcières. Les bûchers brûleront à l'ombre de leur trône. Comme on dit le fou, disons la sorcière $d u$ roi. La frontière qu'elle marque est plus inquiétante encore que la folie.

\footnotetext{
56. Athalie, v. 3-4.

57. Athalie, v. 347.

58. Athalie, v. 662.

59. Athalie, v. 1815.

60. Athalie, v. 1242.

61. R. Muchembled, op. cit., p. 33.
} 